\title{
Città del Vaticano, Biblioteca Apostolica Vaticana, Vat. gr. 1094
}

\author{
I.-II. Costantinopoli, XIV sec. (ante 1378).
}

Comp., ff. A, 1-227, A'; num. a penna nel marg. sup. est. di ogni foglio; sul f. A è annotata la segnatura del codice e il contenuto Disputationes contra Palamam hereticum. Sono bianchi i ff. 109v e 111v. Legatura in pelle bianca con segnatura del codice crisografa impressa sul dorso.

\section{I. ff. 1-204 (Constantinopoli, XIV sec.)}

\begin{abstract}
Cart.; fil. ff. 2, 4, 5, 7, 9, 12, 13, 16, 18, 19, 22, 23, 113, 115, 116, 118, 120, 123, 124, 127, 128, 131, 135, 137, 138, 141, 142, 145, 146, 149, 150, 154, 155, 157, 159, 161, 163, 166, 169, 171, 172, 174, 176, 179, 183 , 184, 187, 188, 190, 195, 196, 197, 198, 201, 202, 205 simile a fruit (M/Tr nr. 4398, 1368), ff. 33, 36, 37, 40, 64, 66, 69, 71, 72, 79, 81, 83, 84, 86, 99, 100, 105, 107, 108, 110 simile a fruit (M/Tr nr. 4326, 1360/1370), ff. 26, 28, 29, 31, 50, 51, 54, 55, 56, 58, 61, 63, 73, 74, 77, 90, 94, 103, 224, 225 simile a cloche (M/Tr nr. 2950, XIV m.), ff. 41, 43, 46, 48 simile a $\operatorname{arc}(\mathrm{M} / \mathrm{Tr} \mathrm{nr} .362,1360 / 1370) ; 1-7^{8}(56), 8^{8-1}$ (63), 9-208 (175), $21^{8-2}(191), 22^{6}(197), 23^{6+1}(204)$; num. fasc. nel marg. inf. est. del primo foglio di ogni fascicolo a partire dal secondo fasc., numerati da $\beta^{\prime}$ a kү'; mm $228 \times 145=24 / / 167 / / 36 \times 20$ // 100 // 25; a piena pagina, 11. 25-26, unità di rigatura mm 5 (f. 26); rigatura assente.
\end{abstract}

\section{Scrittura}

Nel codice si individuano due mani principali: Anonimo $\alpha$ (ff. 1-63r, 1. 6, 64r-109r,

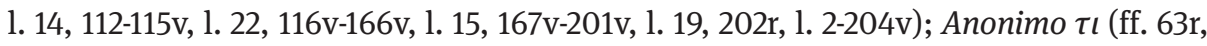
1. $7-63 v)$.

\section{Decorazione}

Anonimo $\alpha$ ha vergato in rosso le iniziali minori e i tituli delle opere e dei paragrafi.

\section{Interventi successivi}

In questa unità intervengono altre due mani: <Giovanni Ciparissiota > ha corretto le proprie opere in alcuni punti; ha inserito i titoli vergati in rosso nel marg. sup. est. di alcuni fogli (ff. 1r, 11v, 25v, 38r, 44r, 79v, 90v) e alcune integrazioni testuali (ff. 48v, 87v, 95r, 195v, 199r); <Isacco Argiro> ha integrato nuove porzioni di testo su fogli origina- 
riamente bianchi (ff. 110r-111r, 115v, 1. 23-116r, 166v, 1. 16-167r) o ha aggiunto note a margine (ff. 194v-195rv, 201v, 1. 20-202r).

\section{II. ff. 205-227 (Costantinopoli, XIV sec.)}

Cart.; fil. ff. 207, 209, 212, 215, 217, 218, 220 simile a cercle (M/Tr nr. 2161, 1360/1370); 24-26 (221), $27^{6}(227)$; num. fasc. nel marg. inf. fino a $\kappa \zeta^{\prime} ; \mathrm{mm} 229 \times 142=24 / / 168 / / 36 \times 18 / / 113 / / 31$; a piena pagina, ll. 30-31/rr. 30, unità di rigatura mm 4 (f. 110); tipo S/L 00D1(Muz 1-1/0/0/J); tracciate sul recto le linee di giustificazione e sul verso le rettrici.

\section{Scrittura}

Un solo copista: <Isacco Argiro (cf. Polemis 2012, LV, senza l'indicazione dei fogli)> (ff. 205-227).

\section{Contenuto}

Giovanni Ciparissiota, Libri octo criminationibus Palamae respondentes, Liber III

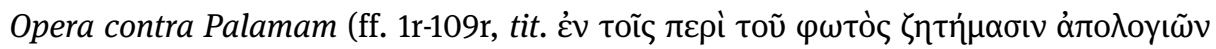

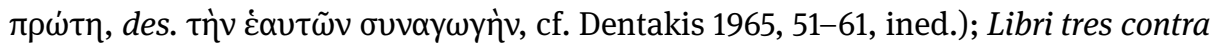
Palamitarum apostasian Liber IV Opera contra Palamam (ff. 110r-226v, tit. $\pi \rho 0 \theta \varepsilon \omega \rho i \alpha$

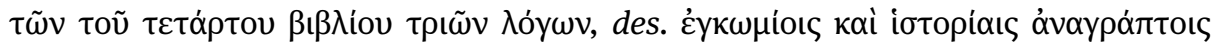

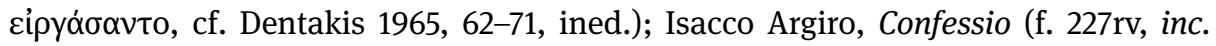

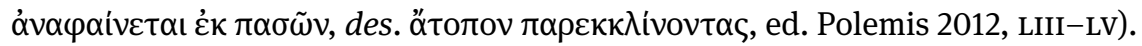

\section{Relazioni stemmatiche}

Il Vat. gr. $1094\left(\mathrm{~V}_{1}\right)$ è uno dei quattro testimoni di XIV secolo delle opere di Giovanni Ciparissiota. Il codice trasmette, insieme al Par. gr. 1246 (P) e al Laur. Plut. $8.8\left(\mathrm{~L}_{1}\right)$, le otto Apologie contro Palama e i Tre libri contro l'apostasia dei palamiti. Il fatto che Giovanni Ciparissiota, come nel Vat. gr. 704 (V), sia intervenuto nei margini correggendo e ampliando il proprio testo in più punti consente di riferirne la realizzazione entro il terzo quarto del XIV secolo (terminus ante quem 1378, anno della morte di Ciparissiota). La preghiera presente al f. 227 e attribuita da Polemis a Giovanni Ciparissiota (Polemis 2012, LIII) va più plausibilmente attribuita ad Argiro, che ha scritto una preghiera del tutto simile a questa in altre due sue opere: nel cap. 21 dell'Adversus Cantacuzenum (Vat. gr. 1096) e nella lettera a Gedeone Zografo (cf. Vat. gr. 1102). 


\section{Storia del codice}

Il codice è presente nella Biblioteca Apostolica Vaticana dal pontificato di Sisto IV (1471-1484): è infatti registrato nell'inventario redatto nel 1475 dal bibliotecario Bartolomeo Sacchi detto il Platina e oggi conservato nel Vat. lat. 3954, f. 73r, n. 700 Palame adversariorum disputationes.

\section{Bibliografia}

\section{Cataloghi}

Devreesse (1965) 75, 173, 228, 302, 334, 376, 398; Lilla (2004) 5-6.

\section{Edizioni}

Dentakis (1964); Dentakis (1965); Polemis (2012).

\section{Codicologia e paleografia}

Mercati (1931) 254; Meyendorff (1959); Polemis (2012) LV, LVI n. 80, LVII n. 82, LX n. 84.

\section{Riproduzioni}

Dentakis (1965) tav. XI. 